\title{
PROBLEMS WITH FINITE DENSITY SIMULATIONS OF LATTICE QCD
}

\author{
Ian BARBOUR and Nasr-Eddine BEHILIL \\ Department of Physics. The University, Glasgow, G12 8QQ, Scotland \\ Elbio DAGOTTO, Frithjof KARSCH, Adriana MOREO, Michael STONE and H.W. WYLD \\ University of Illinois at Urbana-Champaign, Department of Physics, Loomis Laboratory, \\ 1110 W. Green St., Urbana, IL 61801, USA \\ Received 2 April 1986

\begin{abstract}
We present a discussion of problems that have arisen in attempts to understand the behavior of lattice QCD at high densities. The effects observed in the lattice simulations do not seem to be consistent with what we expect from the usual ideas of chiral symmetry breaking. In particular, at zero quark mass, there does not seem to be a massive baryon at finite density.
\end{abstract}

\section{Introduction}

The picture of hadrons provided by QCD implies that there should be a number of interesting effects observed in nuclear matter at high temperature and pressure: one expects both deconfinement, producing a quark-gluon plasma, and a restoration of the chiral symmetry whose breaking is a characteristic of the low-energy interactions of nucleons. These effects might be observed in high-energy heavy-ion collisions and in astrophysical phenomena such as neutron stars [1].

It is possible to study both these effects numerically in lattice simulations of QCD. Including nonzero temperature in the simulations is quite easy since one merely makes a virtue of the finite extent of the lattice, and there have been many studies of these transitions both using the "quenched" approximation and with dynamical fermions [1,2]. There have also been a number of theoretical papers addressing the problem of high density by including a nonzero chemical potential. The earliest of these addressed the basic methods of adding a chemical potential on the lattice [3-5] while more recent papers have been using standard mean field techniques to analyze the chiral restoration transition [6,7]. Relatively few numerical simulations have been published, however $[3,7-9]$. This is probably not due to a lack of effort - but more due to puzzlement over the interpretation of the results, as they do not fit in with expectations. 
In the case of the gauge group $\mathrm{SU}(2)$ the puzzle is transient: a detailed analysis at nonzero $\mu$ shows that the most straightforward mean field ansatz is incompatible with the Monte Carlo simulations (both quenched and unquenched), but in this case it is possible to obtain agreement with a much more refined ansatz which takes into account the degeneracy of the baryons and mesons and the possibility of the vacuum realigning in the presence of baryonic matter [7].

The real problems begin with the larger nonabelian groups. Monte Carlo simulations for $\mathrm{SU}(N), N>2$ gauge theories are difficult due to the appearance of a complex fermion determinant $[9,10]$. However this does not seem to be the major problem. Mean-field as well as (quenched) Monte Carlo calculations seem to indicate that, contrary to expectations, QCD at zero temperature and finite density has either a lowest baryon excitation level with a mass given by $m_{\text {baryon }}=\frac{3}{2} m_{\pi}$ or a state of quark matter which has a bulk energy per baryon corresponding to this mass. Thus it seems that, in the limit of zero quark mass, there is no massive baryon at finite $\mu$ on the lattice. This paper is devoted to discussing calculations that lead us to this puzzling state of affairs.

The first section will review the effects of including a chemical potential for the Kogut-Susskind [6] fermions, the effects we expect to see and a general outline of what is actually seen in the computer simulations. In the second section we will explain, in terms of the eigenvalues of the lattice Dirac operator, how the lattice simulation produces the apparently unphysical effects. In the third section we present some of the data we have obtained and illustrate the different approaches we have taken to the problem. Finally in the last section we speculate on whether we are seeing lattice artifacts, a serious breakdown of our understanding of lattice QCD or, more interestingly, real physics.

\section{Finite chemical potentials}

The most convenient way to generate an ensemble of quarks or nuclei at high density is to add a chemical potential for the quarks. We can include a chemical potential in our simulations by a simple expedient $[3,4]$ of providing an $\exp (\mu)$ factor for the links in the positive imaginary time direction and an $\exp (-\mu)$ factor for those links going backwards. The lagrangian for staggered (Kogut-Susskind) fermions then reads:

$$
S_{\mathrm{f}}=m \sum_{x} \bar{\psi}_{x} \psi_{x}+\sum_{x, \nu} \bar{\psi}_{x} D_{x y}^{v} \psi_{y}
$$

with

$$
\begin{aligned}
& D_{x y}^{i}=\frac{1}{2} \eta_{i}(x)\left[U_{x, i} \delta_{y, x+i}-U_{y, i}^{\dagger} \delta_{y, x-i}\right], \\
& D_{x y}^{0}=\frac{1}{2}\left[\mathrm{e}^{\mu} U_{x, 0} \delta_{y, x+0}-\mathrm{e}^{-\mu} U_{y, 0}^{\dagger} \delta_{y, x-0}\right] .
\end{aligned}
$$


Here $\mu$ is the chemical potential, $m$ is the quark mass, the lattice spacing $a$ has been set to unity, and the rest of the notation is standard. This procedure has a number of advantages over other possibilities $[5,12]$ - in particular the derivative of the free energy with respect to $\mu$ yields the correct conserved current for all values of $\mu$. If we work on a lattice of infinite extent in the time direction - i.e. at zero temperature - the inclusion of $\mu$ should make no difference provided it is small enough; all the world lines of the particles will be finite loops and so the factors of $\exp (\mu)$ from one side of the loop will exactly cancel those on the other. In general we expect that, as long as the chemical potential is smaller than the mass of the lowest-lying baryonic state divided by the number of quarks in the baryon, all physical observables agree with their $\mu=0$ value. In particular the number density $\nu$, and energy density $\varepsilon$

$$
\begin{gathered}
n=\frac{\partial}{\partial \mu} \ln Z \\
\varepsilon=\left\langle\operatorname{Tr} D^{0}\left(m+\sum_{\nu} D^{\nu}\right)^{-1}\right\rangle-\frac{1}{4}\left(N-m\langle\bar{\psi} \psi\rangle_{T=0}\right)
\end{gathered}
$$

vanish for $\mu<\mu_{0}$, with $\mu_{0}$ defining the threshold for baryon production. How this works on the lattice is illustrated for the free theory $(U=1)$ in fig. 1 . We show the number density for various values of the bare mass. The number density vanishes up to a critical value of the chemical potential given by:

$$
\mu_{0}=\ln \left(m+\sqrt{1+m^{2}}\right) \text {. }
$$

This is the mass of a free fermion on a lattice as determined by the location of the pole in the lattice propagator. A similar result holds for the energy density. Notice there appears a second "threshold" which corresponds to the filling of all states in the first Brillouin zone. In the free theory the critical value for this largest nontrivial chemical potential is given by:

$$
\mu_{1}=\ln \left(\sqrt{m^{2}+d-1}+\sqrt{d+m^{2}}\right)
$$

$d$ denoting the space-time dimension. (A consistency check for the mass of the particle that has been created at $\mu_{0}$ and starts contributing to the thermodynamics can be obtained by calculating the ratio $E / n$.) Thus the finite density formulation on the lattice seems to work as expected for the free theory.

Another case where these ideas can be tested explicitly is the simple U(1) gauge theory. The U(1) theory has no centre and hence has only meson states comprising correlated quark-antiquark world lines. Slicing the system across any $t=$ constant hypersurface always reveals the same number of quarks and antiquarks (this 


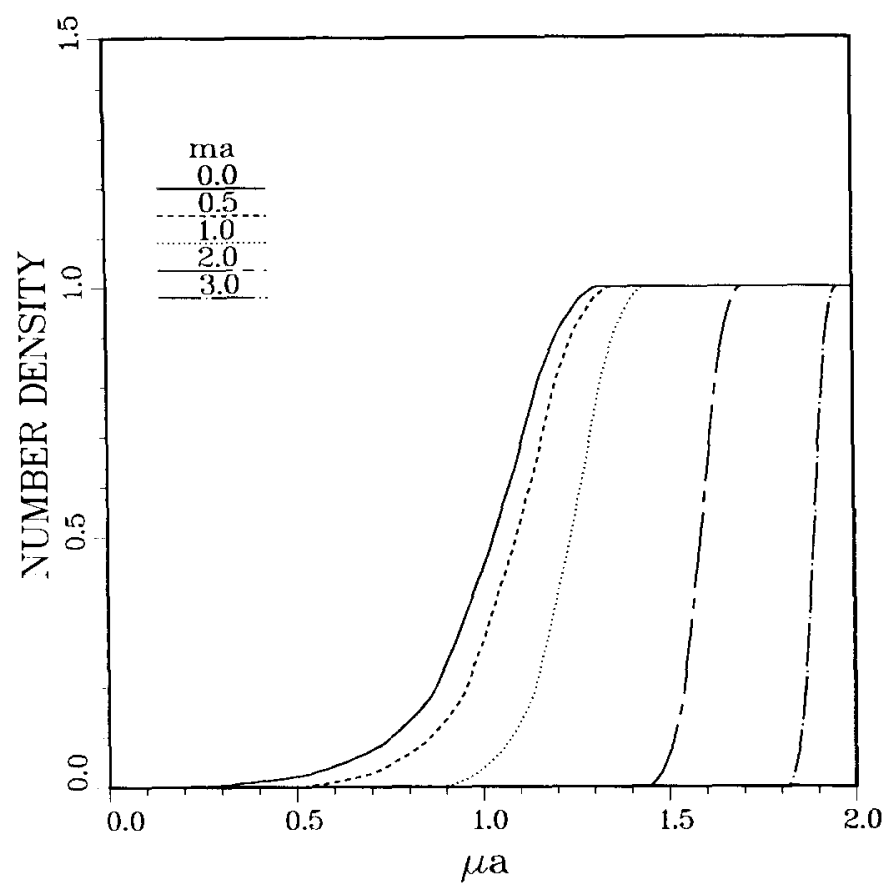

Fig. 1. Number density versus chemical potential $\mu$ for the free Fermi gas at zero temperature on an infinite lattice. Shown are results for different bare masses as indicated in the figure.

remains true even in the weak coupling, deconfined phase because gauge invariance requires electrical neutrality) so the theory should be $\mu$ independent. This is one of the few cases where our theory agrees with the simulations: the $U(1)$ output is indeed $\mu$ independent - indeed it has to be in any of the $\mathrm{U}(N)$ groups because we can always absorb the $\exp (\mu)$ factor into the radius of the group manifold without changing anything in the sums we are performing.

The difficulties begin with the nonabelian groups whose nontrivial centres allow the existence of various gauge singlet "baryon" states in addition to the mesons. It is these that we expect to produce by the addition of the chemical potential. If we are in a confining phase the particles produced will be baryons consisting of three ( $N$ for $\mathrm{SU}(N)$ ) quark world lines bound together with an effective chemical potential $3 \mu$. As $\mu$ is increased there will be a value for $\mu$ such that $3 \mu$ will equal the baryon mass. Any increase beyond this will lead to it being favourable for long loops to wind right around the lattice in the time direction so there is now a finite density of baryons in the system. (One might worry that there would immediately be an infinite density of such loops - indeed there would be if the particles were free bosons - but the Pauli principle is enforced by choosing antiperiodic boundary conditions and so the density remains finite; the Fermi sea just fills up to $E=3 \mu$.) 
This observation should give a simple way of determining the baryon mass without having to construct operators coupling to the baryons or obtain baryon propagators: just observe the value of $\mu$ at which effects begin to be seen in any observable, multiply by three and we should have the mass of the lowest-lying state with baryon quantum numbers ${ }^{\star}$. Unfortunately this simple scenario does not seem consistent with the results obtained from actual computer simulations.

From the discussion in the previous paragraphs we would expect that physical observables are $\mu$ independent up to some critical value of $\mu$ which is related to the threshold for baryon production. When one looks at plots of $\langle\bar{\psi} \psi\rangle$ at fixed quark mass $m$ and different values of $\mu$ one sees, indeed, that $\langle\bar{\psi} \psi\rangle$ stays constant for a while and then goes to zero with increasing $\mu$ as expected [3], but as $m$ is decreased the value of $\mu$ at which the baryon number density becomes nonzero and $\langle\bar{\psi} \psi\rangle$ begins to change also goes to zero. This would seem to imply zero baryon mass. This pattern of behaviour might be expected for the gauge group SU(2), where the baryon is a boson in the same multiplet as the pion and thus has a mass which tends to zero as the square root of the quark mass, but is hardly to be expected in SU(3) where we expect the baryon mass to tend to a fixed, nonzero, value as $m \rightarrow 0$. In fact the data are relatively insensitive to the gauge group (we will discuss results for $S U(2), S U(3)$ and $S U(4)$ gauge groups) and all seem to coincide with the $S U(2)$ picture in that we begin to see effects at a value of $\mu$ which is one half of the Goldstone meson mass [7]; it is as if the meson were a two-quark state and not a quark-antiquark state. There are also un-nerving consequences for the chiral symmetry breaking in the theory. When $\mu=0$ plots of $\langle\bar{\psi} \psi\rangle$ against mass show convincing discontinuities at $m=0$ (modulo finite size effects) indicating that chiral symmetry is broken. As soon as $\mu$ is greater than zero, however, the plots of $\langle\bar{\psi} \psi\rangle$ seem to vary smoothly with $m$ so that the chiral symmetry is restored for arbitrarily small $\mu$. (It is possible to convince oneself otherwise, as some of us did in earlier work [3], but the arguments of the next section will make the existence of any discontinuities for $\mu \neq 0$ very unlikely.) Again this is not what one expects on the basis of massive nuclei where a critical density must be exceeded before the symmetry is restored.

Our first suspicions about these anomalies were that they were artifacts of the quenched approximation for the fermions. Without fermion loops the theory is not unitary and therefore it is possible that different ways of obtaining the baryon mass could be inconsistent; certainly only a subset of the particle world lines (those corresponding to cyclic permutations of initial and final particle locations [3]) are included in measurements of the baryon density and $\langle\bar{\psi} \psi\rangle$. Recently we have obtained data with dynamical fermions included using the complex Langevin

\footnotetext{
* This is strictly only true if we ignore the nuclear binding energy and the Fermi motion of the nuclei. What we are actually making is a sample of nuclear matter at the lowest energy that can exist in equilibrium with the vacuum. For real nuclear matter the energy per baryon is only tens of $\mathrm{MeV}$ different from the baryon mass.
} 
algorithm and they show exactly the same anomalies. Thus something is seriously wrong with the finite $\mu$ calculations.

\section{Eigenvalue distributions}

A way of understanding the computer output, if not the physics behind it, is given by looking at the distribution of eigenvalues of the lattice Dirac operator in background gauge fields obtained either from just the pure gauge action ("quenched" case) or with the fermion determinant included ("dynamical fermions"). The usual massless Kogut-Susskind lattice Dirac operator is skew-hermitian and has purely imaginary eigenvalues $i \lambda_{n}$, in terms of which $\langle\bar{\psi} \psi\rangle$ is given by:

$$
\langle\bar{\psi} \psi\rangle=\sum_{n} \frac{1}{i \lambda_{n}+m} .
$$

In the infinite volume limit the eigenvalues coalesce to form a cut which provides the discontinuity in $\langle\bar{\psi} \psi\rangle$ at $m=0$ :

$$
\langle\bar{\psi} \psi\rangle_{m=0+}-\langle\bar{\psi} \psi\rangle_{m=0-}=2 \pi \rho(0),
$$

where $\rho(\lambda)$ is the eigenvalue density. If we include a nonzero value for $\mu$ the skew-hermiticity is spoilt and the eigenvalues move off the imaginary axis. In fact the first-order change is the addition of a purely hermitian operator so that any eigenvalue that moves initially moves off perpendicularly to the imaginary axis:

$$
\Delta \lambda=\Delta \mu\langle J\rangle,
$$

where $\langle J\rangle$, the lattice fermion-number operator evaluated for the eigenvector of interest, is a real number of $\mu=0$. It is possible for an eigenvalue to not move if the eigenvector is localized, as the introduction of $\mu$ can be gauged away in return for the introduction of discontinuity conditions at the imaginary time boundaries. Clearly a localized eigenvector will not extend across the boundaries at low temperature and is therefore insensitive to such boundary conditions. We hope that the eigenvectors are not localized, however, as such states do not satisfy the chiral Ward identities in a way that gives rise to the usual Goldstone theorem [13].

In practice evaluation of the eigenvalue distribution by use of the Lanczos algorithm [14] shows that, at all couplings, and even at very low temperature (i.e. long lattices in the time direction) all the eigenvalues move off axis to form a roughly uniform strip whose width increases monotonically with $\mu$. Eventually for larger $\mu$ the eigenvalues form a band, leaving the region around $\lambda=0$ completely depleted of eigenvalues. This behavior is shown in fig. 2a-e. For the following discussion the structure of the eigenvalue distribution on the real axis is important. We see from fig. 2 that $\lambda_{\max }=\max \mid \lambda_{\mathfrak{\Im} \lambda=0}>0$ for all positive $\mu$ and that $\lambda_{\min }=$ $\min |\lambda|_{\{\jmath=0}>0$ provided that $\mu>\mu_{1}$. From the eigenvalue distribution we deduce $\mu_{1}=0.5 \pm 0.05$ at $\beta=0$. The maximal and minimal eigenvalues on the real axis are directly related to the behaviour of the physical observables. For bare masses $m>\lambda_{\max }$ all observables will agree with their $\mu=0$ values, while for $m<\lambda_{\min }$ all 

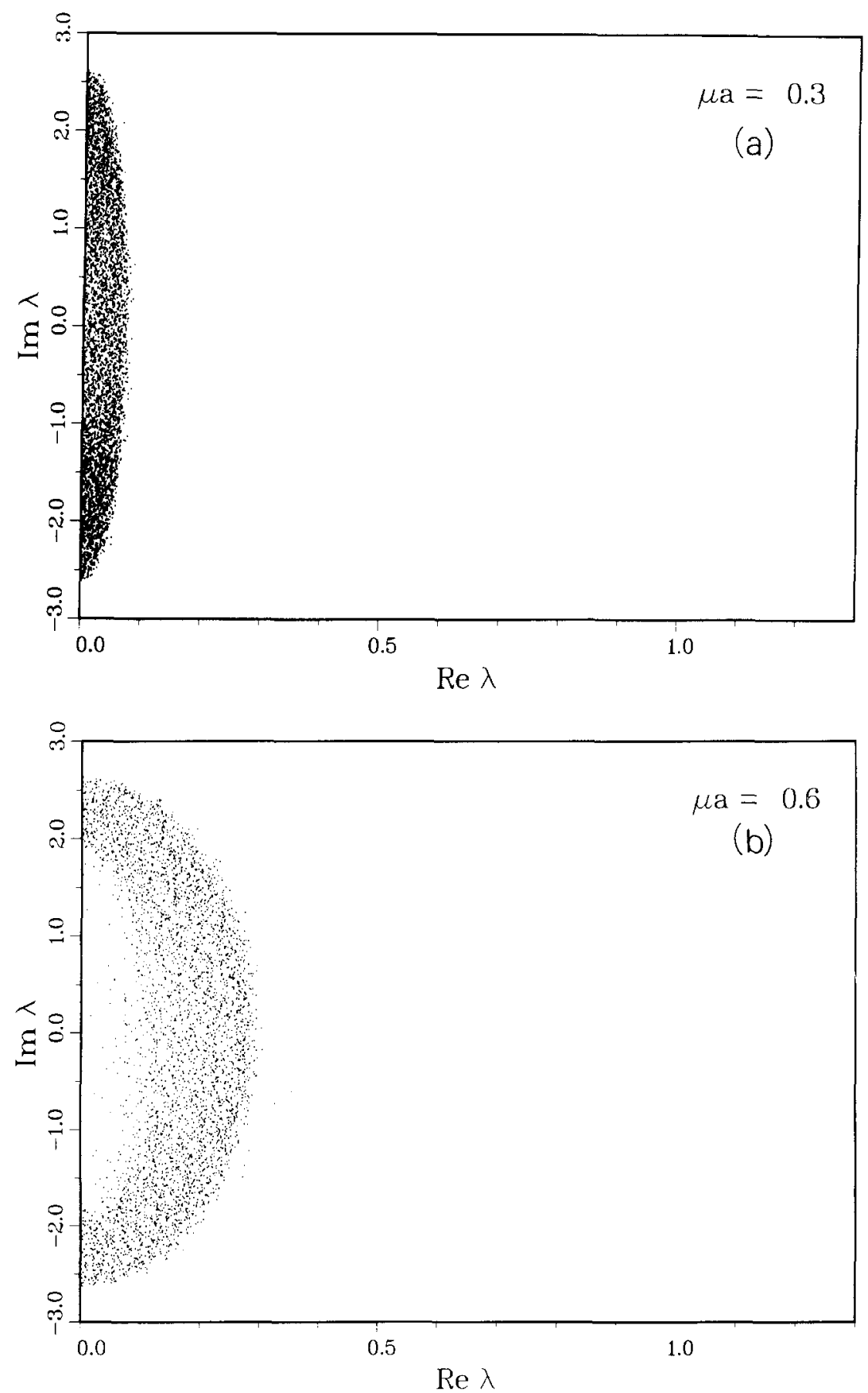

Fig. 2. The distribution of eigenvalues $\lambda$ of the Dirac matrix for staggered fermions at $\beta=0$. Shown are the eigenvalues obtained from 6 random gauge configurations ( $\beta=0$ quenched) on a $4^{3} \times 8$ lattice for different values of the chemical potential, $\mu=0.3$ (a), 0.6 (b), 0.9 (c), and 1.2 (d). 

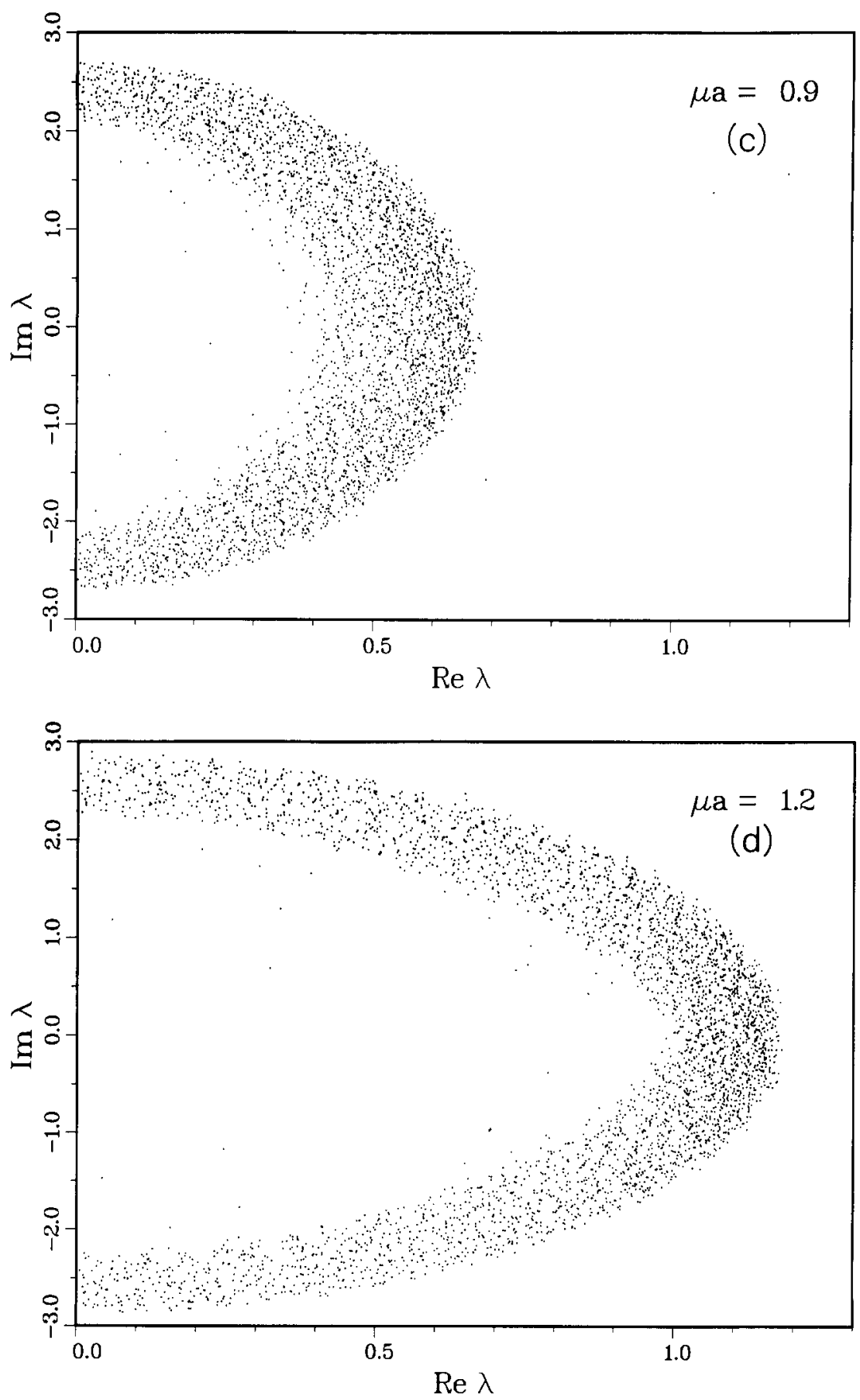

Fig. 2. (continued) 

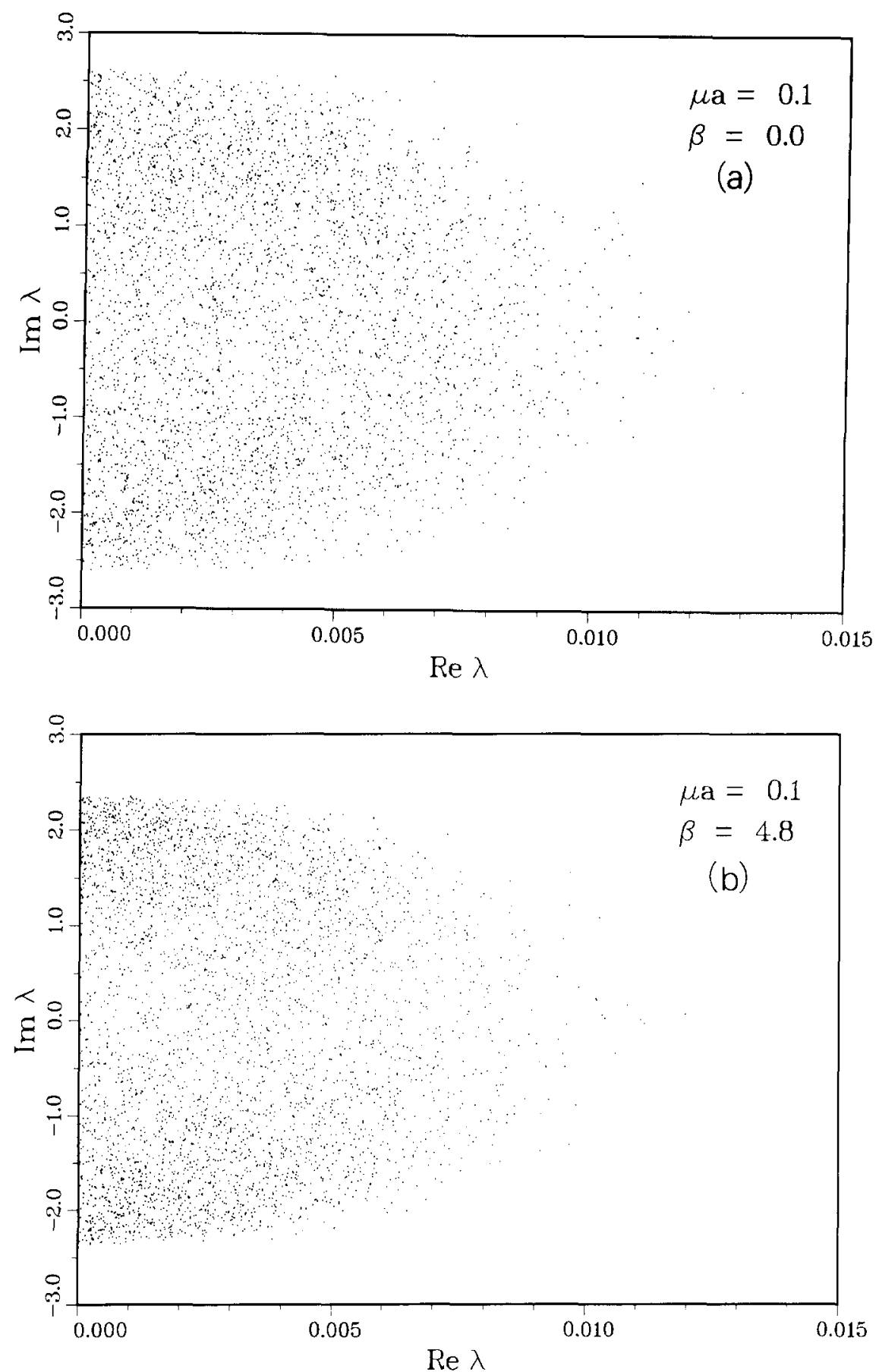

Fig. 3. Same as fig. 2 but for fixed chemical potential $\mu=0.1$ and various values of $\beta=0.0$ (a), 4.8 (b), $5.2(\mathrm{c}), 5.6(\mathrm{~d})$, and $6.0(\mathrm{e})$. 

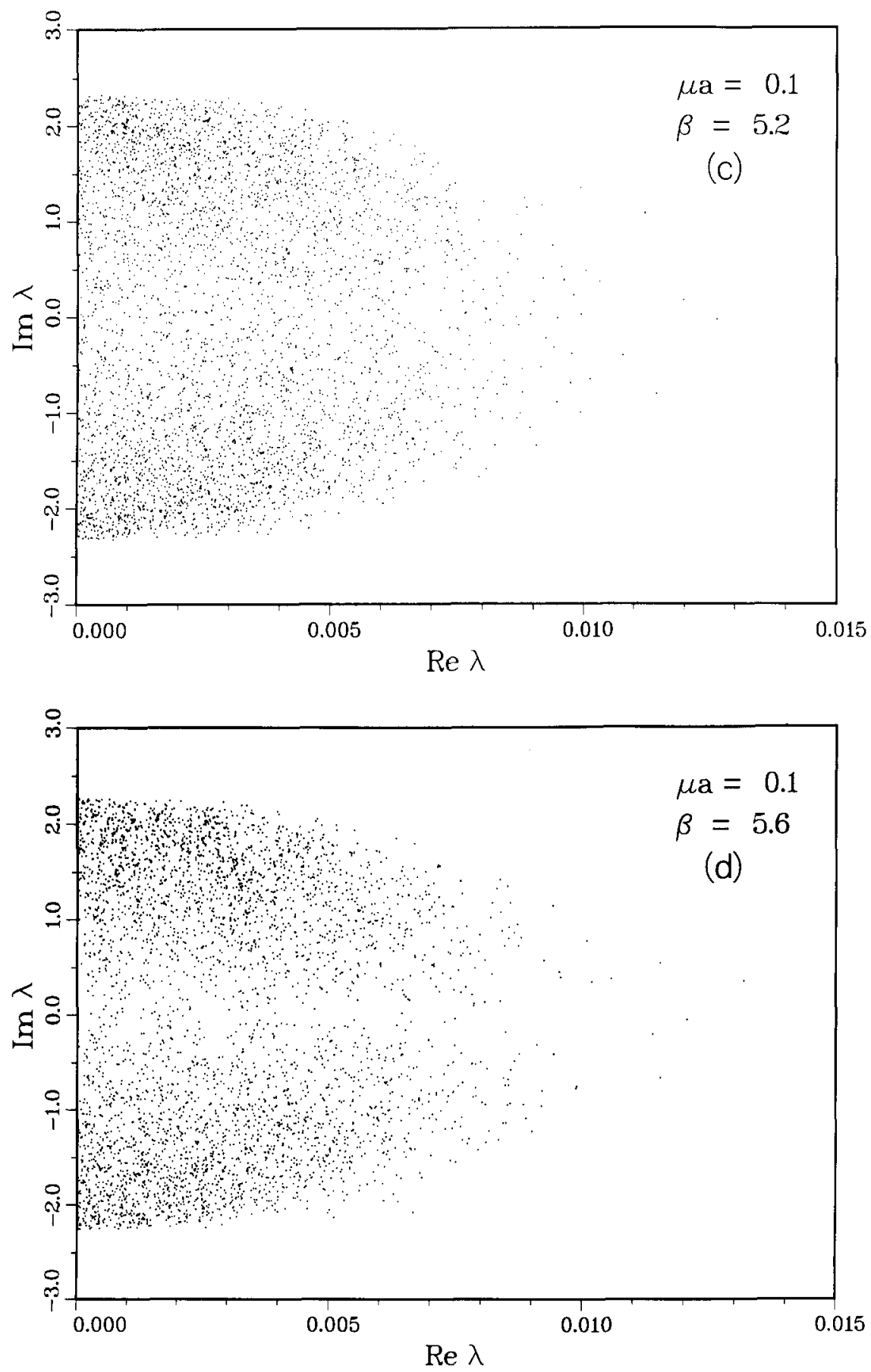

Fig. 3. (continued) 


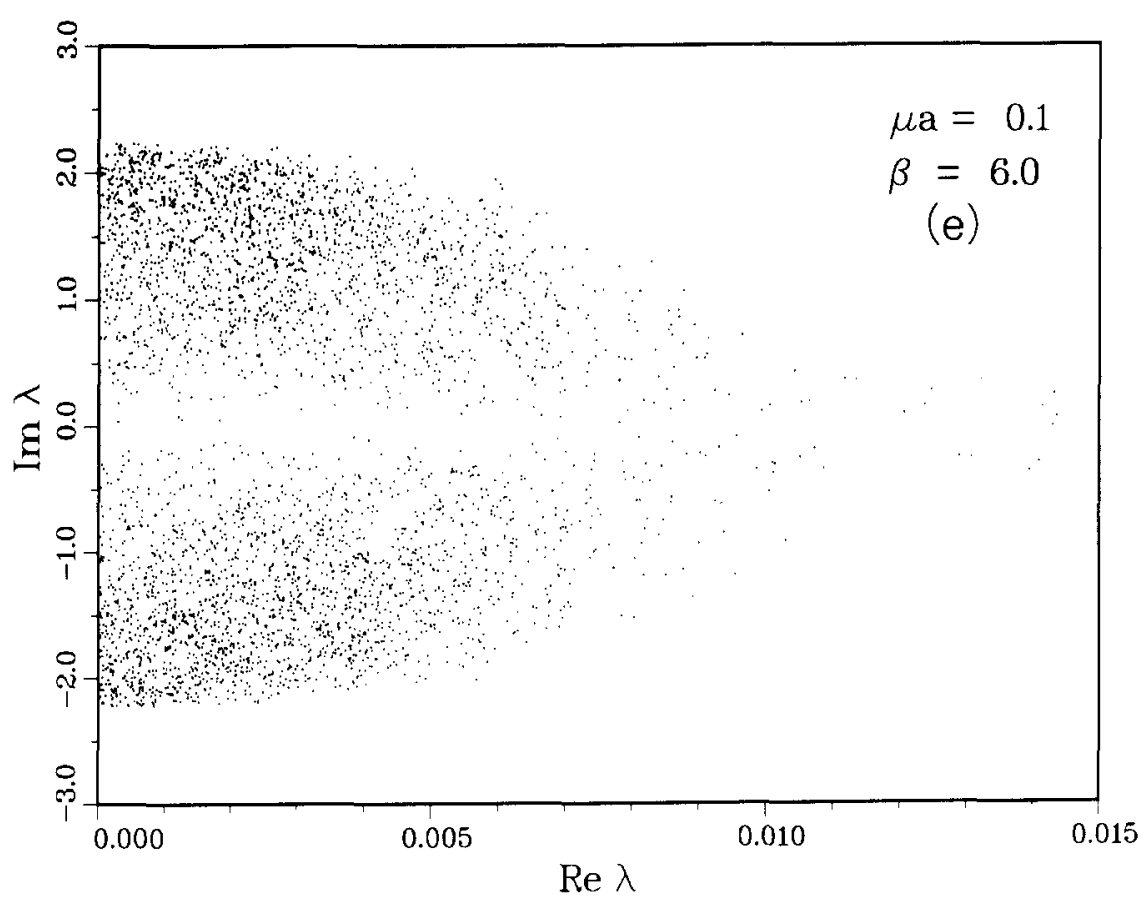

Fig. 3. (continued)

allowed states in the first Brillouin zone are occupied and all observables have reached their limiting, high density, values. In particular $\langle\bar{\psi} \psi\rangle=0$ for all $m<\lambda_{\min }$. Thus in order to be sensitive to the chiral limit we should discuss what happens for $\mu \leqslant \mu_{1}$, i.e. in the case where $\lambda_{\min }=0$. For all $\mu \leqslant \mu_{1}$ there is a uniform band of eigenvalues. There is no sign of any residual singular distribution on the imaginary axis. This is the numerical source of the problems we saw in the previous section. If we use the quenched case to start with, a strip of eigenvalues with no delta function concentration on the imaginary axis cannot give rise to a discontinuity in $\langle\bar{\psi} \psi\rangle$. One can easily see this by considering an electrostatic analogy for the two-dimensional complex plane: a pole can be thought of as a charge, and the values of a function expressed as a sum of poles are the electric field produced by the two-dimensional charge distribution. A delta-function charge density produces an abrupt change in the field as one passes through it while a smooth charge distribution can only cause a smooth variation in the field. Thus $\langle\bar{\psi} \psi\rangle$ goes continuously through zero as $m$ changes sign, even in the infinite volume limit. Because of this we find chiral symmetry breaking disappears for arbitrarily small $\mu$. Similarly the fact that physical effects are observed for small $\mu$ as $m \rightarrow 0$ means that the lowest mass state containing a net number of fermions (i.e. a quark or baryon but not a meson which does not see $\mu$ ) is becoming massless as the quark mass is reduced to zero. This appears to happen for $\operatorname{SU}(N), N=2,3,4$.

In fig. 3 we show eigenvalue distributions for a fixed value of $\mu=0.1$ and various values of $\beta$. We see that the general pattern discussed above for $\beta=0$ persists at 
finite $\beta$. The eigenvalues start moving away from the real axis (this is partly a finite temperature effect [15]); however, they still occupy a strip in the complex plane. We thus conclude that the above observations are valid for a coupling range reaching up to $\beta=6.0$. We will return to a discussion of the weak coupling regime in the next section.

We may hope to be rescued from this dilemma by the introduction of dynamical fermions - but in fact they exacerbate the problem. The inclusion of the fermion determinant means that we no longer have a fixed eigenvalue distribution for all $\mathrm{m}$. We must consider the distribution to depend on the current value of the mass - but for each fixed mass eq. (3.1) still gives $\langle\bar{\psi} \psi\rangle$. The fermion determinant is just:

$$
\operatorname{Det}\left(\sum_{\nu} D^{\nu}-m\right)=\prod_{n}\left(\lambda_{n}^{2}-m^{2}\right)
$$

so that as we vary the mass the eigenvalues are repelled from the location of $m$ and there is even less chance of there being a discontinuity in $\langle\bar{\psi} \psi\rangle$. The apparently vanishing baryon mass will also remain. This pattern of behaviour is insensitive to the gauge group and hence the expected pattern of chiral symmetry breaking ${ }^{\star}$.

\section{The data}

Let us now discuss in some detail the results obtained in Monte Carlo simulations of the SU(3) gauge theory. We have studied the chemical potential dependence of the chiral order parameter, baryon number density and energy density in the quenched approximation. To evaluate all these quantities we require the inverse of the Dirac matrix. We have calculated this using different techniques: pseudofermion methods, the conjugate-gradient algorithm as well as exact inverses, obtained using LINPAC routines, on small lattices. All approaches give results consistent with one another. The problems we are worrying about in this paper can most convincingly be seen in the strong coupling limit. Of course we are aware that we should go to the weak coupling regime to make contact with continuum physics. However, the strong coupling limit has the advantage that we can compare results with analytic calculations; meson and baryon masses are well known [17] and the effect of virtual quark loops is expected to be small.

According to the discussion in the previous sections we would expect that physical observables agree with their $\mu=0$ values until the chemical potential is

* The fundamental representation of $\mathrm{SU}(2)$ is pseudoreal and, at $\mu=0$, has $\mathrm{U}(2 N)$ chiral symmetry which is expected [16] to break to $\mathrm{Sp}(N)$ with the baryons being bosons in the same multiplet as the Goldstone boson; $\mathrm{SU}(3)$ should have the familiar pattern of $\mathrm{U}(N) \otimes \mathrm{U}(N)$ breaking to diagonal $\mathrm{U}(N)$ with massive baryons. At strong coupling on the lattice the residual symmetries are different: $N$ flavours of lattice Kogut-Susskind fermions correspond to $4 N$ flavours in the continuum. Thus, for example, if the gauge group is $\mathrm{SU}(2)$ with the quarks in the fundamental (pseudoreal) representation, the continuum symmetry is $\mathrm{U}(8 N)$ which should break to $\operatorname{Sp}(4 N)$. The lattice symmetry is $\mathrm{U}(2 N)$ and in strong coupling it breaks to $\mathrm{O}(2 N)$. There is probably no contradiction since $\operatorname{Sp}(4 N)$ has a natural $\mathrm{O}(4 N)$ subgroup which can contain the $\mathrm{O}(2 N)$ and so the full $\mathrm{Sp}(4 N)$ can be obtained in the continuum limit. 

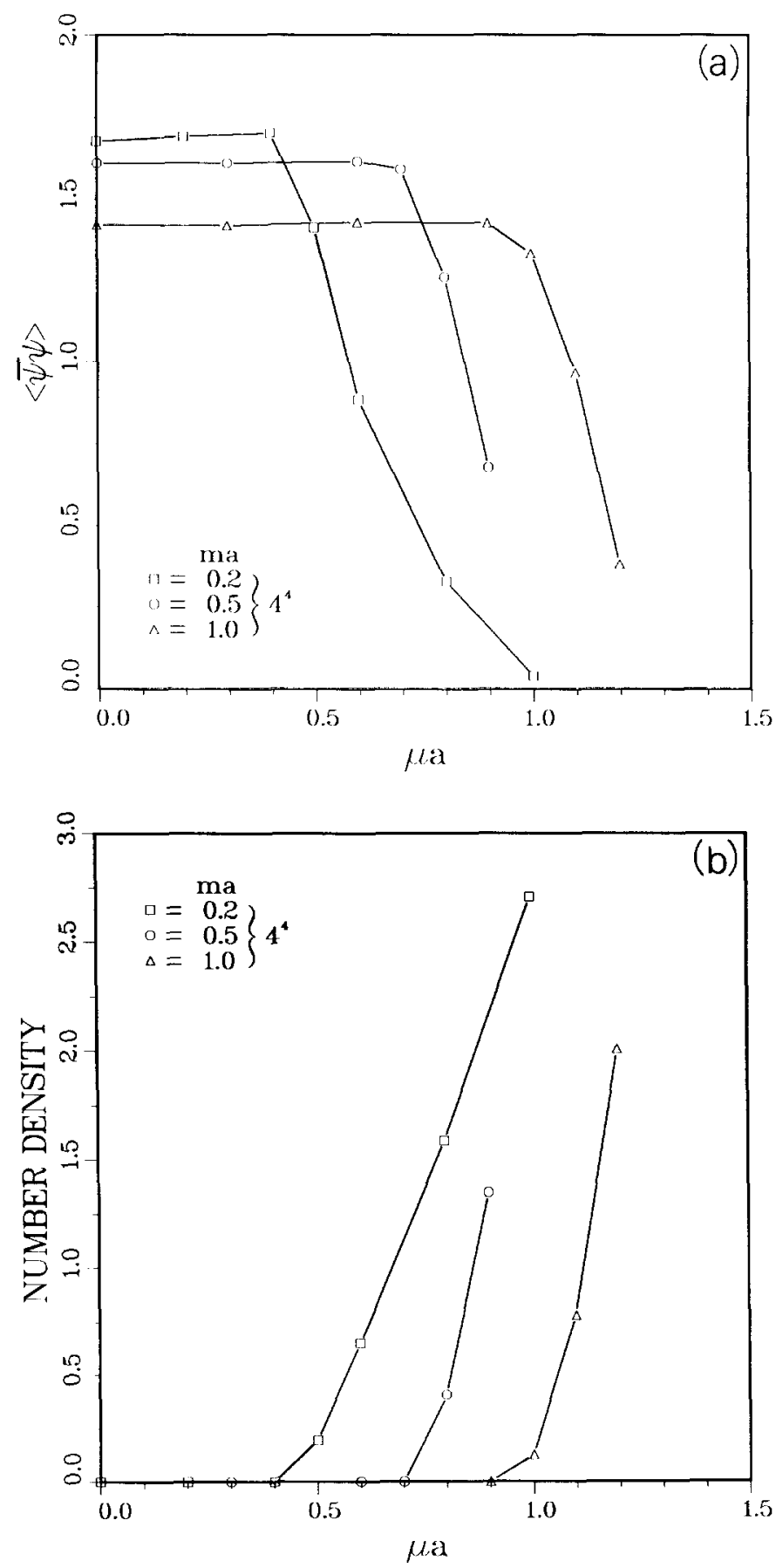

Fig. 4. (a) The chiral order parameter, $\langle\bar{\psi} \psi\rangle$ of the SU(3) gauge theory with staggered fermions versus chemical potential $\mu$ on a $4^{4}$ lattice at $\beta=0$ and various values of the bare mass, $m=0.2,0.5$ and 1.0 .

(b) Same as fig. 4 a but for the baryon number density. (c) Same as fig. 4 a but for the energy density. 


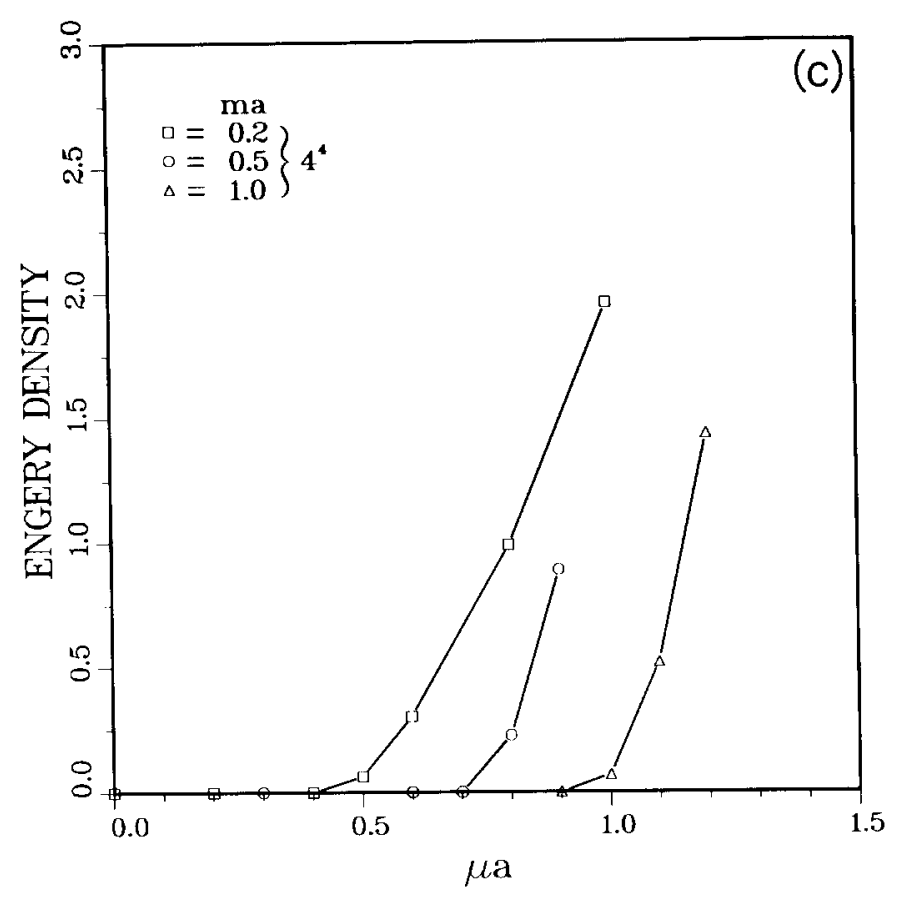

Fig. 4. (continued)

larger than the threshold for baryon production. In the strong coupling limit the baryon mass in the staggered fermion formulations is given by [17]

with

$$
m_{\text {baryon }}=\ln \left[\frac{1}{2} c^{3}+\sqrt{1+\frac{1}{8} c^{6}}\right]
$$

$$
c=\left(m+\sqrt{2 d+m^{2}}\right) .
$$

Thus the critical $\mu$ should be larger than

$$
\mu_{0}=\frac{1}{3} m_{\text {baryon }}>1.0 \ldots .
$$

In fig. 4 we show results for $\langle\bar{\psi} \psi\rangle$, the baryon number density and the energy density obtained from 50 random configurations $(\beta=0)$ on a $4^{4}$ lattice using the pseudofermion method [18] to invert the Dirac matrix. One clearly sees that for all three values of the bare quark mass $m a=0.2,0.5,1.0$, the energy and number density become nonzero for values of the chemical potential well below the threshold for baryon creation. To rule out a failure of the pseudofermion method we repeated the calculation with an algorithm which inverts the matrix exactly. The results obtained from 50 configurations agree well with the pseudofermion results. In fig. 5 we show $\langle\bar{\psi} \psi\rangle$ obtained from the exact inverse. It shows similar behavior. $\langle\bar{\psi} \psi\rangle$ breaks away from its $\mu=0$ value much earlier than expected. (Fig. 5 also shows a few data points from $6^{3} \times 12$ and $12^{4}$ lattices using the conjugate gradient 


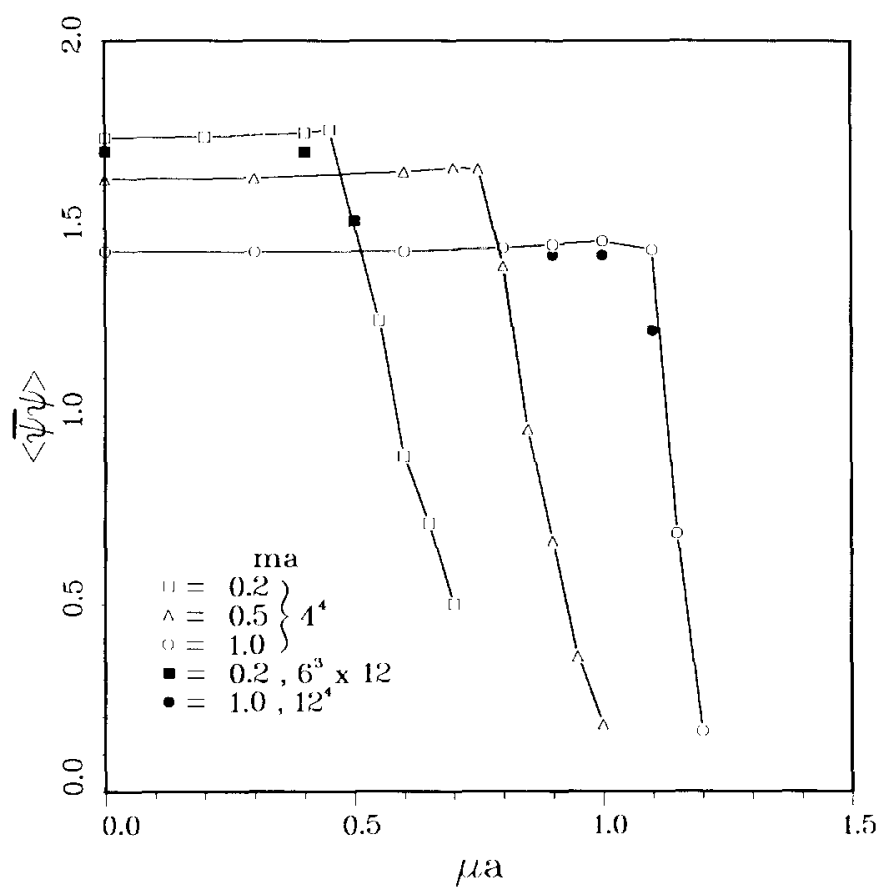

Fig. 5. Same as fig. 4a, however an exact inverse of the Dirac matrix has been used to calculate $\langle\bar{\psi} \psi\rangle$. Also shown are conjugate gradient results obtained on large lattices of size $6^{3} \times 12$ and $12^{4}$.

algorithm to calculate $\langle\bar{\psi} \psi\rangle$. These data show that finite size effects are small at strong coupling.) We found that the critical value of $\mu$ is in agreement with the lowest meson mass:

$$
\mu_{0}=\frac{1}{2} m_{\pi}
$$

with the pion mass given by [17]:

$$
m_{\pi}=\ln \left[1+\frac{1}{2}\left(c^{2}-2 d\right)+\sqrt{\left(c^{2}-2 d\right)+\frac{1}{4}\left(c^{2}-2 d\right)^{2}}\right] .
$$

This suggests that a baryonic excitation far below the baryon mass exists, having a mass 1.5 times the meson mass. When the quarks are massless we find thus a massless baryon at finite $\mu$. In addition the data suggests that for $m=0,\langle\bar{\psi} \psi\rangle$ vanishes for all nonzero $\mu$.

In fig. 6 we show the phase diagram emerging from these simulations with staggered fermions as well as the eigenvalue distributions. Beside the baryon threshold and the anticipated phase boundary defined by eq. (4.5) we also show the result of a mean field calculation for the critical line. This calculation has been performed in analogy to the calculation presented in ref. [7] for the SU(2) case. (Some details of the specific SU(3) problems are discussed in the appendix.) It is interesting to see that also the mean field calculations seem to indicate that the 


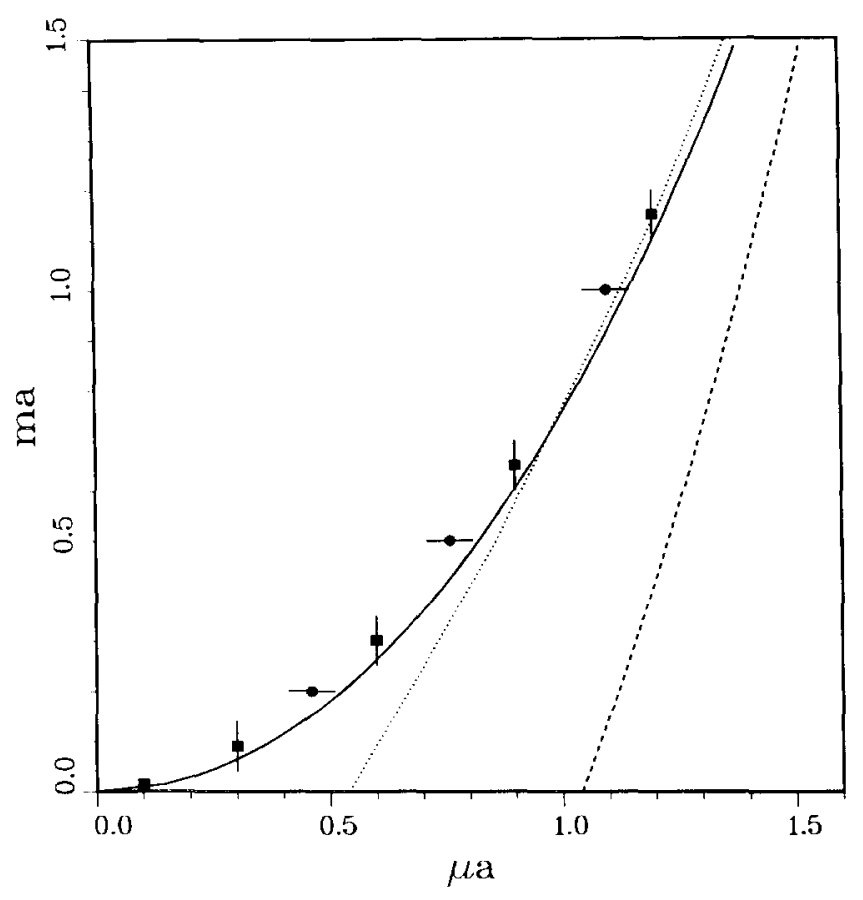

Fig. 6. Phase diagram in the mass-chemical potential plane of the $S U(3)$ gauge theory with staggered fermions in the strong coupling $(\beta=0)$ limit. The data points show the threshold value where physical observables start deviating from their $\mu=0$ values. They have been obtained from the exact inversion results shown in fig. $5(\bullet)$ and from the Lanczos calculation shown in fig. 2 (ם). Also shown is the expected critical line corresponding to the baryon threshold (-) and the pion threshold $(-)$ which seems to describe the Monte Carlo data. The dotted curve is the result of the mean field calculation discussed in the appendix.

critical value of the threshold for baryon creation is determined by the pion mass, at least for intermediate masses, rather than the expected baryon mass. At small masses the mean field results give a finite critical value which seems to be consistent with the value $\mu_{1}$ found from the eigenvalue distribution as critical value for filling all states of the Brillouin zone. One other substantial difference between the mean field and the Monte Carlo data is in the order of the transition which is first order for the mean field but apparently second order for the Monte Carlo for any finite quark mass ${ }^{\star}$. This is not unusual as mean field calculations often get the orders of transitions wrong, but it does lead to a difference in the interpretation of the physics as we will discuss in the last section. The degree of agreement between the two methods does suggest, however, that the inclusion of dynamical fermions would not alter the results of the quenched approximation. (In the SU(2) case the quenched

* At infinite mass the system becomes essentially one-dimensional and we will find a first-order phase transition. This is consistent with the eigenvalue distribution which becomes a band of zero width on a unit circle. See also fig. 1 for the large mass limit in the free theory. 


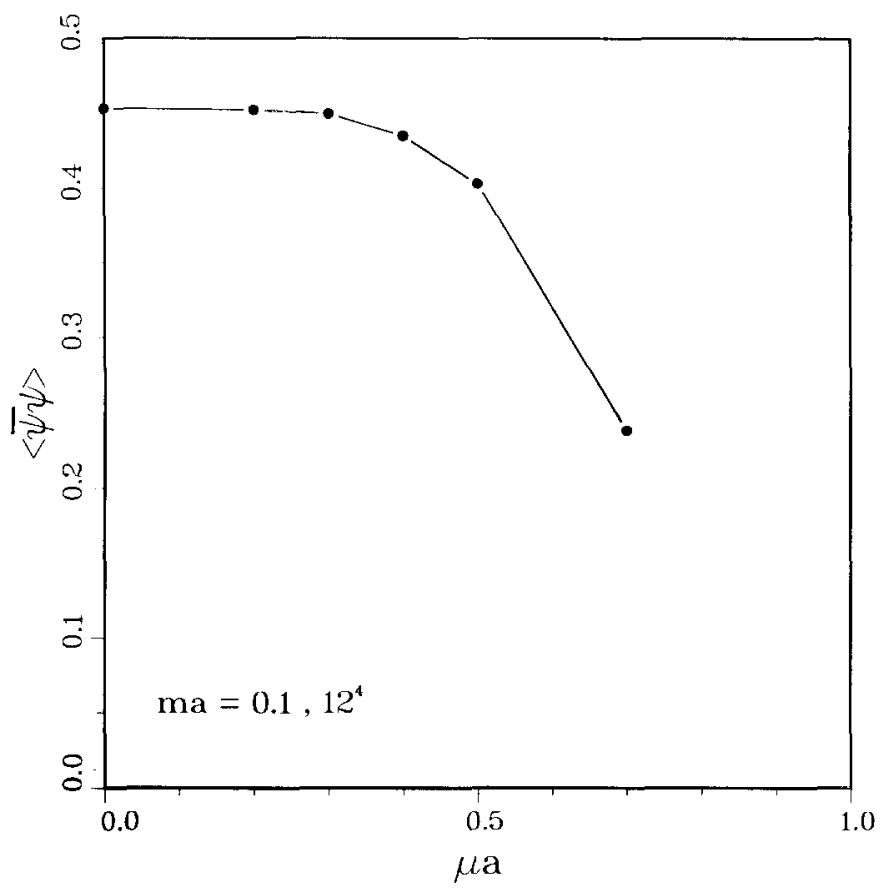

Fig. 7. The chiral order parameter versus chemical potential $\mu$ obtained from conjugate gradient calculations at $\beta=6.0, m=0.1$ on a $12^{4}$ lattice.

and unquenched simulations gave results identical within error bars. For SU(3) we performed some unquenched calculations for a few values of $\mu$ and $m$ using the complex Langevin algorithm $[10,19]$ on a $4^{4}$ lattice and they also agreed with the quenched data.)

Finally to check that we did not just see an artifact of the strong coupling limit we also did a simulation on a $12^{4}$ lattice at $\beta=6.0$. At this coupling the baryon and meson masses have been determined for staggered fermions of bare mass ma= $0.1,0.2,0.3[20]$. We can use these data to check whether the critical $\mu$ is related to the pion mass or the baryon threshold. From ref. [20] we find $\frac{1}{2} m_{\pi}=0.395(5)$ and $\frac{1}{3} m_{\text {baryon }}=0.50(3)$ for a quark mass of 0.1 . Notice that in contrast to the strong coupling regime these two possible critical values are now quite similar. This makes it difficult to ultimately rule out that the baryon mass triggers the onset of thermodynamics in the continuum regime of the lattice theory. Much smaller quark mass values would be necessary to get firm results. This, however, would also require better values of the hadron masses on larger lattices. Our results, obtained with the conjugate gradient algorithm, for this bare mass value are shown in fig. 7 . They seem to support that also at $\beta=6.0$ the threshold is driven by the pion.

We also verified that this behavior does not seem to be due to the fermionic nature of the massive baryon in SU(3): a simulation for quenched SU(4) showed a behavior analogous to the ones discussed here for $\mathrm{SU}(3)$. 


\section{Speculations}

There are various possible explanations for the peculiar behavior we see in these simulations. An error in our codes is unlikely since we have evaluated the quantities concerned in a number of different ways and with programs written independently by several people. We could be seeing artifacts due to the method of introducing the chemical potential, but this is unlikely since the $\exp (\mu)$ procedure can be thought of in various ways and in all of them the connection with the baryon mass seems inevitable. Other procedures for the introduction of the chemical potential $[1,12]$ will suffer from the same problems since they are essentially the same except for a redefinition of $\mu$ and they all coincide for small $\mu$.

We could be seeing other lattice artifacts coupled with finite size effects. We have run on fairly large lattices and this does not seem to change the results - but consider the following scenario: The chemical potential method finds the energy of the lowest state with net quark quantum numbers. When the baryon mass is measured by other methods one usually sets out to measure the energy of the zero momentum state on the plausible grounds that it is the one of lowest energy and thus it is this energy that is equal to the mass. It may be that lattice systems contain states with nonzero momentum, baryon quantum numbers and energy smaller than the $k=0$ states, so it would be these unexpected states that we are producing. This does not happen in the simple large $d$ or large $N$ expansions for the baryons (although it does happen for all the mesons except the $\pi$ ) but it does not seem easy to eliminate this possibility for finite $d$ and $N$. Such lattice artifacts should be absent at weak coupling in the continuum. At weak coupling, however, it is difficult to be at low temperatures and so the real states may be thermally excited. This would mean that our observations may be due to a mixture of unphysical states at strong coupling and finite size effects at weak coupling - and would therefore be absent if we could work on really large lattices at weak coupling. While this is a plausible explanation, in the absence of a better understanding of the underlying dynamics observed in the strong coupling regime, we are, at present, unable to find further support for such a scenario.

One possibility that occurred to us was that we were seeing the effect of many light flavours on the stability of nuclear matter. There has, for some time, been speculation that nuclear matter rich in strange quarks would be more stable than a system of hadrons with the same quantum numbers and density [21] - simply because the more flavours of quarks the less effective is the exclusion principle in pushing up the Fermi energy; the other flavours can occupy the lower energy states. Thus our observations might be real physics. In the strong coupling mean field calculations this is exactly what happens: the system jumps directly from the vacuum to a state at sufficiently high density to have restored the chiral symmetry. The quarks in the plasma have bare or "current masses" instead of the usual "constituent masses", which come from the chiral symmetry breaking. Because of this the resulting "quark nuggets" [21] have an energy per baryon which is much less 


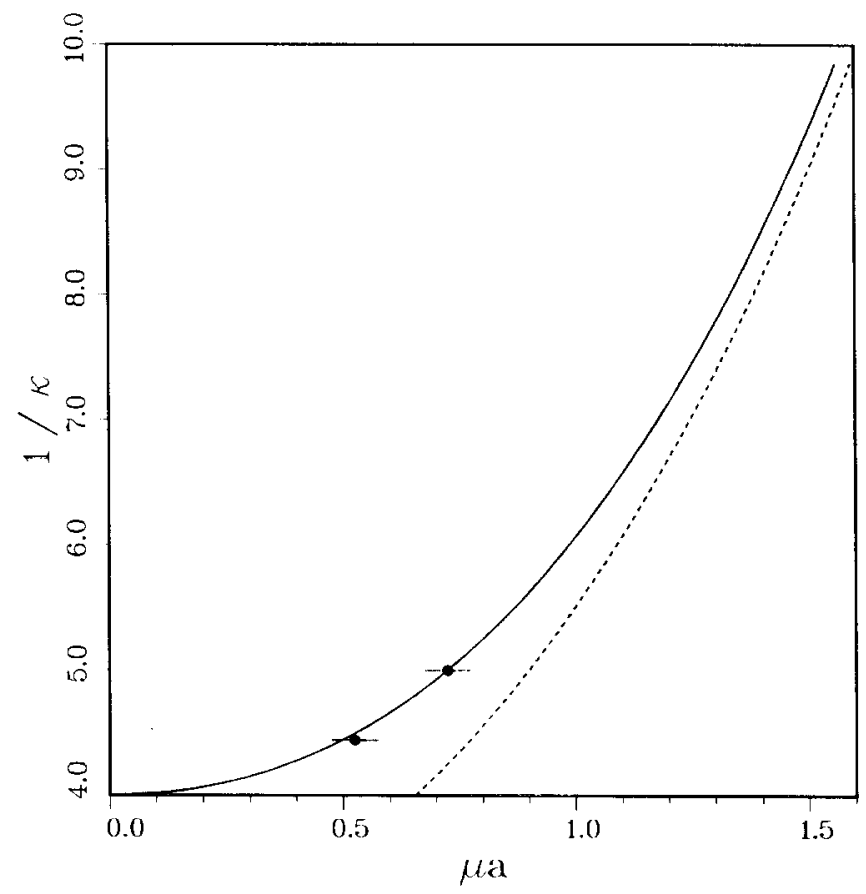

Fig. 8. Phase diagram in the hopping parameter $\kappa$-chemical potential plane of the SU(3) gauge theory with Wilson fermions in the strong coupling $(\beta=0.0)$ limit. The data points have been obtained by using the conjugate gradient method on a $4^{4}$ lattice. Also shown are the critical lines corresponding to the baryon and pion thresholds (notation is the same as fig. 6).

than the usual baryon mass and the vacuum can coexist with these nuggets instead of with ordinary nuclear matter made up of intact baryons. This seems like an intriguing prospect but the Monte Carlo data with staggered fermions do not see the same jump in density that the mean field produces (corresponding to the possibility of a mixed phase of vacuum and nuggets). Also we should be able to remove the effect by running simulations with the physical number of flavours. We have tested this by performing computations with Wilson fermions where, at the expense of explicit chiral symmetry, we can perform simulations with one or two light flavours instead of the multiples of four that we get with Kogut-Susskind fermions. Fig. 8 shows the phase diagram resulting from the runs on small lattices at strong coupling, compared with strong coupling calculations of the baryon and meson masses [22]. Once again we see that we are seeing effects as soon as the chemical potential exceeds half the pion mass so this explanation is probably not viable.

\section{Conclusion}

The basic conclusion of this paper is that there appears to be a problem with the conventional interpretation of simulations of QCD at finite chemical potential. The chiral symmetry is apparently restored at arbitrarily small $\mu$ - long before we would 
expect there to be any effects at all if the baryon has a nonzero mass. The data seem to suggest that either there exist baryonic states which become massless in the zero quark mass limit and have an energy equal to $\frac{3}{2} m_{\pi}$ or stable quark matter with low mass per baryon. Although the results presented here are based on a study of the strong coupling regime using "quenched" Monte Carlo techniques, we think that we have given enough evidence that these results will hold over a wide range of couplings. It may be that deep in the continuum regime $(\beta>6.0)$ the lattice formulation will reproduce the expected continuum, zero temperature, finite density scenario. However, to verify this will require fairly large lattices $\left(\geqslant 12^{4}\right)$ and small quark masses $(m<0.1)$ (in order to create a light pion which really is much lighter than the baryon). It seem to us more promising to try to get a better understanding of the effects observed in the strong and intermediate coupling regime at finite density. In particular it will be crucial for further studies of the finite density theory to understand the significance of the light baryonic states of matter generated at strong coupling.

This work was supported by grants NSF-DMR-84-15063, NSF-PHY-82-01948 and NSF-PHY-83-04872. We acknowledge the support of the National Center for Supercomputing Applications (NCSA) at the University of Illinois where most of the computer simulations at Urbana were done. We would also like to thank, for their patience and help, all the people with whom we have discussed these problems. These include Pedrag Cvitanovic, Eduardo Fradkin, Rajiv Gavai, Peter Hasenfratz, John Kogut, Peter Orland, Janos Polonyi, Steve Shenker, Helmut Satz, Junko Shigemitsu and many others.

\section{Appendix}

In this appendix we briefly describe the calculations leading to the mean field results presented in sect. 4.

Working in the strong coupling limit $\left(1 / g^{2}=0\right)$ the integration over the gauge fields can be done exactly for the groups $\mathrm{SU}(2)$ and $\mathrm{SU}(3)$. For the latter the only integral which had to be evaluated in addition to those given in the literature was one with six matrix elements ( $\left.\int \mathrm{d} U\left[U U U U^{\dagger} U^{\dagger} U^{\dagger}\right]\right)$. Using these the partition function can be rewritten exactly as

$$
\begin{aligned}
Z= & \left(\prod_{x, a=1,2,3} \int \mathrm{d} \bar{\psi}_{x}^{a} \mathrm{~d} \psi_{x}^{a}\right) \exp \left[\frac{1}{12} \sum_{x, \nu} M_{x} M_{x+\nu}-\frac{1}{8} \sum_{x, \nu}\left[f_{x, \nu} \bar{B}_{x} B_{x+\nu}-f_{x, \nu}^{-1} \bar{B}_{x+\nu} B_{x}\right]\right. \\
& +\frac{1}{576} \sum_{x, \nu} M_{x}^{2} M_{x+\nu}^{2}-\frac{5}{576} \sum_{x, \nu} \bar{B}_{x} B_{x} \bar{B}_{x+\nu} B_{x+\nu}
\end{aligned}
$$

where

$$
\begin{aligned}
f_{x, \nu} & =\eta_{\nu}(x), & & \nu=1,2,3 \\
& =\mathrm{e}^{3 \mu}, & & \nu=0
\end{aligned}
$$


and we have defined the composite operators

$$
\begin{array}{ll}
M_{x}=\sum_{a=1}^{3} \bar{\psi}_{x}^{a} \psi_{x}^{a} \quad & \text { (meson) } \\
B_{x}=\psi_{x}^{1} \psi_{x}^{2} \psi_{x}^{3}, & \text { (baryon) }
\end{array}
$$

In a first approximation to eq (A.1) we drop the $M^{2} M^{2}$ and $\bar{B} B \bar{B} B$ terms. It has been proved that this is consistent with a large $d$ (dimension) approach. The $M M$ interaction can be decoupled using the identity,

$$
\exp \left(\frac{1}{12} M_{x} M_{x+\nu}\right)=\iint_{-\infty}^{+\infty} \frac{\mathrm{d}[\Re \sigma] \mathrm{d}[\mathfrak{F} \sigma]}{2 \pi} \exp \left(-\frac{1}{2} \bar{\sigma} \sigma+\sqrt{\frac{1}{24}}\left[\sigma M_{x}+\bar{\sigma} M_{x+\nu}\right]\right),
$$

where $\sigma$ is a complex field attached to the link $(x v)$. In ref. [7] we also used a similar trick for the $\bar{B} B$ interaction because the SU(2) baryon is a boson. However, here the baryon is a fermion and we need Grassmann fields to decouple the interaction. For example we use the identity:

$$
\exp \left[\bar{B}_{x} A_{x}\right]=\int \mathrm{d} \bar{\theta} \mathrm{d} \theta \exp \left[-\bar{\theta} \theta+\bar{\theta} A_{x}+\bar{B}_{x} \theta\right]
$$

where $\bar{B}_{x}$ is an antibaryon at site $x$ and $A_{x}, \bar{\theta}, \theta$ are also Grassmann variables. Now we can easily integrate over the $\bar{\psi}, \psi$ fields. An important difference between SU(2) and $\mathrm{SU}(3)$ is that the latter resulting effective action is quadratic in the fermionic field $\theta$ so we can also integrate it. At the end we arrive at a complicated action for the bosonic field $\sigma$ which is dealt with by the mean field approximation at lowest order. Basically we freeze $\sigma$ to a mean value $\bar{\sigma}$ (proportional to $\langle\bar{\psi} \psi\rangle$ ) which is determined self-consistently. In other words we must minimize the free energy:

$$
\begin{aligned}
F= & \frac{1}{2} \bar{\sigma}^{2}-3 \ln \left(\sqrt{\frac{1}{6}} \bar{\sigma} d\right) \\
& -\frac{1}{2} \int_{0}^{2 \pi} \frac{\mathrm{d}^{d} k}{(2 \pi)^{d}} \ln \left[1+\frac{1}{16 q^{6}} \sum_{j=1}^{d-1} \sin ^{2} k_{j}+\frac{1}{16 q^{6}} \sin ^{2}\left(k_{0}-3 i \mu\right)\right],
\end{aligned}
$$

where

$$
q=m-\sqrt{\frac{1}{6}} \bar{\sigma} d .
$$

The integral in momentum space comes from the nonlocal gaussian integration over the $\bar{\theta}, \theta$ fields. Minimizing eq. (A.6) with respect to $\bar{\sigma}$ we get a result showing a 
phase where $\bar{\sigma}$ is independent of $\mu$ and another phase where $\bar{\sigma}$ is very small for any fermionic bare mass, separated by a line of first-order transitions. The $\overline{\boldsymbol{\sigma}} \approx 0$ phase corresponds to a set of decoupled one-dimensional models as expected in the $\mu=\infty$ limit. The transition line is shown in fig. 6 .

Eq. (A.6) seems rather complicated but in the zero mass limit we can prove that the position of the critical $\mu$ can be obtained easily as follows: Compare the free energies of the one-dimensional model (where only the $\bar{B}_{x} B_{x+0}$ term survives) with the corresponding energy at $\mu=0$ (which is well approximated by keeping just the $M M$ interaction in eq. (A.1)). The result for any dimension is:

$$
\mu_{\mathrm{c}}^{\text {M.F. }}(m=0)=\frac{1}{2} \ln (2 d)-\frac{1}{2}
$$

where $d \rightarrow \infty$ the critical chemical potential tends to one third of the baryon mass, while for $d=4, \mu_{c} \approx 0.54$, in excellent agreement with the complete mean field solution shown in fig. 6 .

We can also repeat the calculation keeping all the terms present in eq. (A.1) by introducing additional decoupling fields. (For example the $M^{2} M^{2}$ term can be decoupled by introducing another bosonic field which we again freeze to a mean-field value etc.) We have explicitly checked that the influence of such additional complications is small.

\section{References}

[1] J. Cleymans, R.V. Gavai and E. Suhonen, Phys. Reports 130 (1986) 219

[2] F. Karsch, Illinois preprint IL-(TH)-86- \#9

[3] J. Kogut, H. Matsuoka, M. Stone, H.W. Wyld, S. Shenker, J. Shigemitsu and D.K. Sinclair, Nucl. Phys. B225 [FS9] (1983) 93

[4] P. Hasenfratz and F. Karsch, Phys. Lett. 125B (1983) 308; H. Matsuoka and M. Stone, Phys. Lett. 136B (1984) 204

[5] N. Bilic and R.V. Gavai, Z. Phys. C23 (1984) 77

[6] E.M. Ilgenfritz and J. Kripfganz, Z. Phys. C29 (1985) 79; P.H. Damgaard, D. Hochberg and N. Kawamoto, Phys. Lett. 158B (1985) 239

[7] E. Dagotto, F. Karsch and A. Moreo, Phys. Lett. 169B (1986) 349

[8] A. Nakamura, Phys. Lett. 149B (1984) 391

[9] J. Engels and H. Satz, Phys. Lett. 159B (1985) 151

[10] F. Karsch and H.W. Wyld, Phys. Rev. Lett. 55 (1985) 2242

[11] J. Kogut, M. Stone, H.W. Wyld, S.H. Shenker, J. Shigemitsu and D.K. Sinclair, Nucl. Phys. B225 [FS9] (1983) 326

[12] R.V. Gavai, Phys. Rev. D32 (1985) 519

[13] A.J. McKane and M. Stone, Ann. of Phys. 131 (1981) 36

[14] I.M. Barbour, J.P. Gilchrist, H. Schneider, G. Schierholz and M. Teper, Phys. Lett. 127B (1983) 433

[15] I.M. Barbour, Proc. Argonne National Laboratory Workshop on Gauge theory on a lattice 1984, eds. C. Zachos, W. Celmaster, E. Kovacs and D. Sivers (Argonne Nat. Lab. Publication CONF8404119)

[16] M. Peskin, in Recent advances in field theory and statistical mechanics, Les Houches session XXXIX, 1982, eds. J.B. Zuber and R. Stora, (North-Holland, 1984) 
[17] H. Kluberg-Stern, A. Morel and B. Peterson, Nucl. Phys. B215 [FS7] (1983) 527

[18] F. Fucito, E. Marinari, G. Parisi and C. Rebbi, Nucl. Phys. B180 [FS3] (1981) 369;

H. Hamber, E. Marinari, G. Parisi and C. Rebbi, Phys. Lett. 124B (1983) 99

[19] G. Parisi, Phys. Lett. 131B (1983) 393;

J. R. Klauder, Stochastic quantization, lectures given at the XXII Schladming School (March 1983)

[20] A. Biloire, E. Marinari and R. Petronzio, Nuc1. Phys. B255 [FS13] (1985) 141

[21] S.A. Chin and A.K. Kerman, Phys. Rev. Lett. 43 (1979) 1292;

G. Baym, E.W. Kolb, L. McLerran, T.P. Walker and R.L. Jaffe, Phys. Lett. 160B (1985) 181;

E. Witten, Phys. Rev. D30 (1984) 272

[22] K.G. Wilson, in New phenomena in subnuclear physics, Erice 1975, ed. A. Zichichi (Plenum, New York 1977);

A. Hasenfratz, P. Hasenfratz, Z. Kunszt and C.B. Lang. Phys. Lett. 117B (1982) 81 